\title{
Environmental Exposures and Hepatocellular Carcinoma
}

\author{
Regina M. Santella ${ }^{1,2}$ and Hui-Chen $\mathrm{Wu}^{1}$ \\ ${ }^{1}$ Department of Environmental Health Sciences, Mailman School of Public Health, Columbia University Medical Center, New York, \\ NY, USA; ${ }^{2}$ Herbert Irving Comprehensive Cancer Center, Columbia University Medical Center, New York, NY, USA
}

\begin{abstract}
Infection with hepatitis $B$ and/or hepatitis $C$ virus is a wellestablished risk factor for the development of hepatocellular carcinoma (HCC). However, it is now clear that certain occupational, environmental, and lifestyle factors also play a role in cancer development. Among these factors are smoking, alcohol consumption, workplace exposure to vinyl chloride, and exposure to polycylic aromatic hydrocarbons and aflatoxins. There is also evidence that several other chemical and infectious agents have a role in inducing HCC in humans. Epidemiologic studies and the use of biomarkers have provided essential data to demonstrate the importance of some of these factors in human risk, while animal studies have suggested that other chemicals may also play a role. Although immunization against hepatitis $B$ virus infection remains the primary method of preventing HCC in regions of the world where this virus is a primary etiologic agent, there is currently no vaccine for hepatitis $C$ virus. Thus, limiting exposure to other known risk factors remains an important mechanism in preventing HCC.

(1) 2013 The Second Affiliated Hospital of Chongqing Medical University. Published by XIA \& HE Publishing Ltd. All rights reserved.
\end{abstract}

\section{Introduction}

Primary hepatocellular carcinoma (HCC) is one of the leading cancers worldwide, with the highest risk in Asia and subSaharan Africa. ${ }^{1}$ The USA, like Europe, is generally considered a low-risk area, but nevertheless HCC in the USA has one of the fastest-growing death rates for any cancer, in both men and women. ${ }^{2,3}$ It is well established that the major risk factor for HCC is chronic infection with hepatitis B or C virus (HBV or HCV) and the resulting cirrhosis. In most parts of the world where HCC incidence is high, HBV is the major risk factor. Chronic HBV infection is believed to be responsible for $55 \%$ of $\mathrm{HCC}$ cases worldwide, and $89 \%$ in regions where the virus is endemic or hyperendemic. ${ }^{4,5}$ In the USA, HCV is a

Keywords: Aflatoxin; Chemical carcinogens; Hepatocellular cancer; Polycyclic aromatic hydrocarbons.

Abbreviations: 4-ABP, 4-aminobiphenyl; $\mathrm{AFB}_{1}$, aflatoxin $\mathrm{B}_{1} ; \mathrm{CI}$, confidence interval; CYP2E1, cytochrome P450 2E1; CSP, Cancer Screen Project; HBsAg, hepatitis B virus surface antigen; HBV, hepatitis B virus; HCC, hepatocellular carcinoma; $\mathrm{HCV}$, hepatitis $\mathrm{C}$ virus; IARC, International Agency for Research on Cancer; OR, odds ratio; $\mathrm{PAH}$, polycyclic aromatic hydrocarbons; RR, relative risk. Received: 12 March 2013; Revised: 29 April 2013; Accepted: 21 May 2013 DOI of original article: 10.14218/JCTH.2013.008XX.

Correspondence to: Regina M. Santella, Department of Environmental Health Sciences, Mailman School of Public Health of Columbia University, 630 West 168th St, New York, NY 10032, USA. Tel.: +1-212-305-1996, Fax: +1-212-305-5328, E-mail: rps1@columbia.edu major risk factor, with approximately $2 \%$ of the population infected. Liver flukes (Opisthorchis viverrini, Clonorchis sinensis) and Schistosoma japonicum, the parasite responsible for schistosomiasis, have also been implicated in the induction of liver cancer (cholangiocarcinoma) in regions where these parasites are common. Human immunodeficiency virus- 1 is another suggested risk factor.

In addition to these infectious agents, a number of chemicals have been implicated in human HCC (Table 1). Perhaps the best documented are aflatoxins, for which biomarkers have provided definitive information on exposure. However, other factors, such as smoking, alcohol consumption, and exposure to vinyl chloride and radioactive compounds, have also been associated with liver cancers. In addition, animal studies have implicated a number of additional chemicals to which humans are sometimes exposed, but for which there are insufficient data for them to be definitively classified as human liver carcinogens. Here, we review some of the data associating induction of human HCC with specific chemical exposures.

\section{Aflatoxins}

Aflatoxins are mycotoxins produced primarily by Aspergillus flavus. Aflatoxin $B_{1}\left(A_{F} B_{1}\right)$ is a potent carcinogen that commonly contaminates foods such as peanuts, corn, and other grains and legumes (reviewed in ${ }^{6}$ ). Once ingested, $\mathrm{AFB}_{1}$ is metabolized in vivo by the cytochrome $\mathrm{P} 450$ system to a highly reactive epoxide that binds to guanine (Fig. 1). This DNA adduct is chemically unstable, and can result in depurination and release of adducts into the urine or opening of the imidazole ring to form a stable ring-opened adduct in the DNA. Replication of damaged DNA, either with apurinic sites or bulky ring-opened adducts, can result in the mutations, translocations, and other alterations associated with HCC development (Fig. 1).

In regions with high $\mathrm{HCC}$ incidence, $\mathrm{HBV}$ infection and $\mathrm{AFB}_{1}$ exposure frequently occur in combination. In addition, it is difficult to accurately determine individual dietary exposure to $A F B_{1}$ from analysis of food because of variations in toxin levels between different batches of food. For these reasons, early studies that carried out dietary surveys produced conflicting results on the role of $\mathrm{AFB}_{1}$ in $\mathrm{HCC}$ risk. The late 1980 s brought the development of biomarkers that could monitor individual exposure to $\mathrm{AFB}_{1}$ by measuring urinary excretion of metabolites and depurinated adducts, DNA adducts in blood or tissue, and protein adducts in blood. There are limited data on the correlation of the various biomarkers of aflatoxin exposure. For example, we have reported a significant association between adduct levels in DNA of tumor tissues and albumin adducts in blood. Mean 
Santella R. M. et al.: Environmental exposures and hepatocellular cancer

Table 1. Agents known/suspected to induce HCC in humans

\begin{tabular}{ll}
\hline Good evidence & Limited evidence \\
\hline Hepatitis B virus & Androgenic steroids \\
Hepatitis C virus & Arsenic and inorganic arsenic compounds \\
Aflatoxin $B_{1}$ & Betel quid without tobacco \\
Alcohol & Polychlorinated biphenyls \\
Cigarette smoke & Trichloroethylene \\
Oral contraceptives & X- and gamma-radiation \\
Plutonium and thorium-232 & \\
Vinyl chloride (liver angiosarcoma) & \\
\hline
\end{tabular}

( \pm standard deviation) levels of $\mathrm{AFB}_{1}$-albumin adducts in individuals with low, medium, and high $\mathrm{AFB}_{1}$-DNA adducts were $51.0 \pm 36.5,70.5 \pm 48.1$, and $84.9 \pm 48.2 \mathrm{fmol} / \mathrm{mg}$, respectively. ${ }^{7}$ In addition, adduct levels in tumor tissue have been found to be higher than in adjacent non-tumor tissues. ${ }^{8}$ The use of these biomarkers in molecular epidemiologic studies has dramatically enhanced our ability to determine the role of aflatoxins in human HCC.

Biomarkers of $\mathrm{AFB}_{1}$ exposure have been used in several different types of epidemiological studies, including ecological, case-control, and prospective nested case-control studies. These studies have been reviewed in detail and here we briefly summarize the findings. ${ }^{9}$ Overall, many ecological studies in Africa and China have found a significant association between dietary $\mathrm{AFB}_{1}$ intake (estimated from contaminated foods) and HCC incidence in several populations, but data on the cross-sectional relationship between urinary $\mathrm{AFB}_{1}$ markers and $\mathrm{HCC}$ incidence are discordant. Our own cross-sectional survey of 250 residents from eight areas of Taiwan with a fourfold variation in HCC mortality found a positive association between urinary $\mathrm{AFB}_{1}$ metabolites and mortality at the township level, and the association was stronger in females with chronic HBV infection than in uninfected females. ${ }^{10}$

Unfortunately, many ecological studies conducted in high HBV-endemic regions have not considered the effect of HBV infection on HCC risk. The reliability of data on HCC incidence and HBV prevalence is limited, because cancer registries and $\mathrm{HBV}$ screening are not nationwide in some countries. Seasonal variations that might affect the levels of<smiles>COc1cc2c(c3oc(=O)c4c(c13)CCC4=O)C1C=COC1O2</smiles>

Aflatoxin B1<smiles>C=C1CCc2c1c(=O)oc1c3c(cc(OC)c21)OC1OC2OC2C31</smiles>

Aflatoxin epoxide<smiles>COCCO</smiles>

Lysine adduct

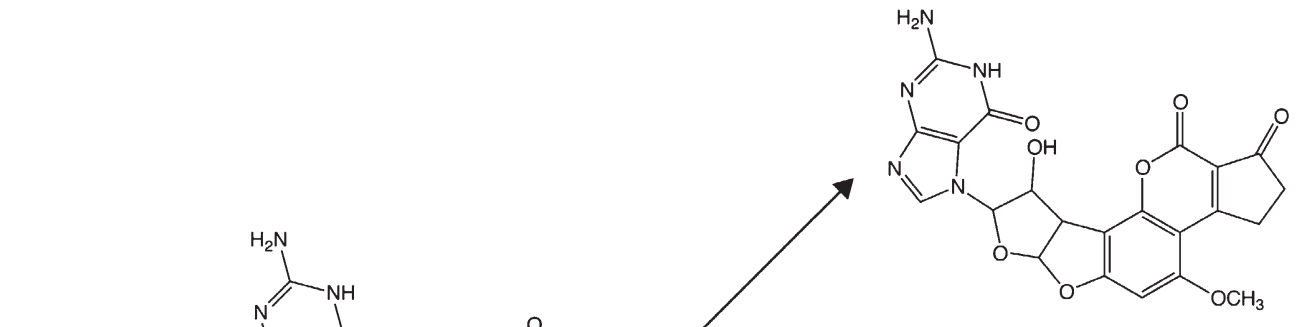

N7 guanine adduct in urine<smiles>COc1cc2c(c3oc(=O)c4c(c13)CCC4=O)C1C(O2)OC([n+]2cn([13C]([O-])O[Na])c(C)c2N)C1O</smiles>

N7 guanine adduct on DNA<smiles>CCC</smiles><smiles>CCC</smiles>

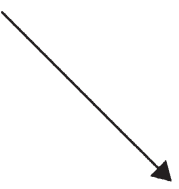

Depurinated DNA

Fig. 1. Metabolism of aflatoxin $B_{1}$ to the reactive epoxide, which can bind with protein to form adducts at lysine residues or react with DNA to form the N7 guanine adduct. The DNA adduct is chemically unstable and can result in depurination of the DNA. The released modified base can be detected in the urine of exposed individuals. A stable ribose ring opened form of the adduct is also seen in low levels (not shown). 
contamination have also not been considered, and some studies have pooled urine samples from individual cases. The lack of individual-level information is a limitation known as the "ecological fallacy", meaning that an association observed between variables on an aggregate level does not necessarily represent the association that exists at the individual level. However, ecological studies can be performed quickly and inexpensively, and have provided some useful information on the association of $\mathrm{AFB}_{1}$ intake and $\mathrm{HCC}$ risk.

Case-control studies in which cases are recruited at the time of disease diagnosis have frequently been used to investigate the relationship between $\mathrm{AFB}_{1}$ exposure and $\mathrm{HCC}$ risk because it is relatively easy to identify cases from hospital data. Although there are conflicting results, dietary data generally do not show an increased risk associated with ingestion of $\mathrm{AFB}_{1}$-contaminated foods. However, dietary questionnaire data are inadequate to measure $\mathrm{AFB}_{1}$ intake because the content of individual foods can vary widely, owing to geographic and seasonal variations. In addition, there may be severe recall bias. $\mathrm{AFB}_{1}$-albumin adducts in blood and $\mathrm{AFB}_{1}$-DNA adducts in leukocytes have been measured in case-control studies. However, these results have also been discordant, with only $\mathrm{AFB}_{1}$-DNA adducts in leukocytes being significantly associated with risk (reviewed in ${ }^{9}$ ). Because subjects were selected at the time of diagnosis, it is unclear whether the cancer status affected biomarker levels. The advantages of these studies are their ability to assess the relationship of $\mathrm{AFB}_{1}$ exposure and $\mathrm{HCC}$ risk at the individual level, and to adjust for potential confounders such as HBV infection. However, all such studies published to date have been small, limiting their ability to draw firm conclusions.

A more valid approach to evaluate the role of $A F B_{1}$ is through prospective studies, measuring levels of $A_{F} B_{1}$ biomarkers in blood/urine specimens collected years before diagnosis. Three major cohort studies have used this study design to address the relationship of $\mathrm{AFB}_{1}$ exposure and HBV infection with HCC incidence. ${ }^{9}$ The first, a nested casecontrol study in a cohort of 18,244 men, was conducted in Shanghai (PR China). ${ }^{11}$ Cases $(n=22)$ were matched with controls for age and area of residence, and $\mathrm{AFB}_{1}$ metabolites and $\mathrm{AFB}_{1}$-N7-guanine adducts were measured. A relative risk (RR) of 3.8 (95\% confidence interval [CI] 1.2-12.2) was observed after adjusting for factors including HBV surface antigen (HBsAg) status, a measure of chronic infection. A strong interaction between $\mathrm{AFB}_{1}$ and $\mathrm{HBV}$ was also reported (RR 60.1; 95\% CI 6.4-561 compared with those negative for $\mathrm{HBV}$ and $\left.A F B_{1}\right)$, although only seven cases and two controls were positive for both $\mathrm{HBsAg}$ and the presence of $\mathrm{AFB}_{1}$ metabolites. ${ }^{11}$ A subsequent follow-up study from this cohort, with a total of 50 cases, showed that the presence of any urinary $\mathrm{AFB}_{1}$ biomarker significantly predicted liver cancer (RR 5.0; 95\% CI 2.1-11.8). ${ }^{12}$

Our Cancer Screen Project (CSP) recruited individuals aged 30-65 years living in several rural townships of Taiwan, where HBV infection is hyperendemic. ${ }^{13-16}$ Preliminary results from this prospective cohort, limited to residents of Penghu Island, who have the highest $\mathrm{HCC}$ mortality and $\mathrm{AFB}_{1}$ exposure in Taiwan, demonstrated a 5.5-fold increased risk of $\mathrm{HCC}$ in individuals with detectable $\mathrm{AFB}_{1}$-albumin adducts. ${ }^{13}$ The entire cohort included 12,024 men and 13,594 women; 56 of these individuals developed HCC during the first 3-4 years of follow-up, and 230 HCC cases were identified after 12 years. ${ }^{14,16}$ Analysis of urine and albumin samples resulted in an RR of 1.5 (95\% CI 1.0-2.6) for $\mathrm{AFB}_{1}$-albumin adducts and 1.8 (95\% CI 1.2-2.6) for AFB $_{1}$ metabolites. ${ }^{16}$ We also observed a fourfold increased risk of HCC for HBsAg-negative individuals with higher levels of urinary $\mathrm{AFB}_{1}$ metabolites $(\geqslant 77 \mathrm{fmol} / \mathrm{ml}){ }^{16}$

In the first (smaller) study, we reported an 111-fold increased risk of $\mathrm{HCC}$ among HBsAg carriers with high urinary $\mathrm{AFB}_{1}$ metabolites and a 70-fold increased risk for detectable $\mathrm{AFB}_{1}$-albumin adducts, compared with those with low/undetectable levels and negative for HBsAg. ${ }^{14}$ In the later study, with a much larger sample size, the effect of combined $A F B_{1}$ exposure and HBV infection was more consistent with an additive than with a multiplicative model; the odds ratio (OR) for those with $\mathrm{AFB}_{1}$-albumin adducts above the mean and presence of HBV was 10.38 (95\% CI 5.73-18.82) compared with those negative for HBV and with adducts below the mean. ${ }^{16}$ Similarly, for those with urinary $\mathrm{AFB}_{1}$ metabolites above the mean and who were HBV positive, the OR was 15.13 (95\% CI 7.83-29.25).

Another study that we conducted in Taiwan, with 4,841 male government workers with chronic HBV infection, has provided additional evidence for the role of $\mathrm{AFB}_{1}$ intake in the development of HCC. ${ }^{17,18}$ After 5 years of follow-up, we observed a dose-response relationship between HCC and serum level of $\mathrm{AFB}_{1}$-albumin adducts ${ }^{17}$ and urinary $\mathrm{AFB}_{1}$ metabolites ${ }^{18}$ among those who had null genotypes of GSTM1 and GSTT1, two genes involved in detoxifying reactive intermediates, but not among those who had non-null genotypes. In a cohort of Chinese men with chronic HBV infection followed for 10 years, the RR of HCC was also significantly increased in subjects with detectable aflatoxin $\mathrm{M}_{1}$ levels. ${ }^{19}$

Overall, prospective studies have shown a strong association between biological markers of $\mathrm{AFB}_{1}$ exposure and risk of subsequent $\mathrm{HCC}$. The interaction between $\mathrm{AFB}_{1}$ exposure and HBV infection on HCC risk has been replicated in different cohorts. However, earlier studies, including ours, suggested a synergistic effect, while our more recent study suggested an additive interaction model. The primary reason for these discrepant results may be the small sample sizes in earlier studies. The fraction of HCC cases attributable to aflatoxin exposure has been estimated to be $4.6-28.2 \% .^{20}$

The importance of aflatoxin exposure on $\mathrm{HCC}$ incidence in the USA is unknown. Analysis of $\mathrm{AFB}_{1}$-albumin adducts in healthy US subjects residing in Texas found detectable levels in $21 \%$ of participants. ${ }^{21}$ We found detectable levels of $A_{F B}$ DNA adducts in three of 19 tumor tissues from individuals in Texas and Louisiana, and five sera from individuals from three different states were positive for albumin adducts. ${ }^{22}$

\section{Alcohol consumption}

Alcohol consumption and the resulting cirrhosis have long been recognized as risk factors for HCC development, with the International Agency for Research on Cancer (IARC) indicating a causal relationship in $1988 .{ }^{23}$ Alcohol is metabolized by alcohol dehydrogenase and cytochrome P450 2E1 (CYP2E1) to acetaldehyde (Fig. 2, reviewed in ${ }^{24}$ ). While alcohol dehydrogenase is the primary enzyme responsible for the metabolism of alcohol, chronic alcohol ingestion results in induction of CYP2E1 and increased metabolism by this pathway. Acetaldehyde is capable of damaging DNA, producing several different types of mutagenic lesion. ${ }^{24}$ In addition, the CYP2E1 pathway also results in free-radical production. 
Santella R. M. et al.: Environmental exposures and hepatocellular cancer<smiles>CCO[C@H](C)[C@H](O)OC=C(C)O</smiles>

$\mathrm{ADH}$ alcohol dehydrogenase

ALDH aldehyde dehydrogenase

Fig. 2. Metabolism of alcohol, first to acetaldehyde and then to acetic acid.

Thus, chronic alcohol ingestion results in both DNA adducts and oxidative damage (Fig. 2).

A number of epidemiologic studies have demonstrated that high alcohol consumption is associated with increased $\mathrm{HCC}$ risk (reviewed in ${ }^{25}$ ). For example, in one study in Italy, among those without HBV or HCV infection, an alcohol intake of $>60 \mathrm{~g} /$ day was associated with an OR for HCC of 7.0 (95\% CI 4.5-11.1) compared with those with an intake of $\leqslant 60 \mathrm{~g} /$ day. ${ }^{26}$ Compared with subjects with low alcohol intake and who were negative for viral infection, those with high alcohol consumption and HCV (OR 109, 95\% CI 50.9-233.0) or HBV (OR 48.6, 95\% CI 24.1-98.0) infection were at dramatically increased risk.

\section{Cigarette smoking}

Cigarette smoking has been extensively investigated in epidemiologic studies of HCC. The results have been somewhat inconsistent, but suggest a small increase in risk. A 2009 meta-analysis identified 38 cohort studies and 58 casecontrol studies on liver cancer and cigarette smoking. ${ }^{27}$ Compared with never-smokers, the adjusted meta-analysis RR for liver cancer was 1.51 (95\% CI 1.37-1.67) for current smokers and 1.12 (95\% CI $0.78-1.60)$ for former smokers. The increased risk among current smokers was consistent across different regions, study designs, study sample sizes, and publication periods. A positive dose-response trend was also observed for the number of cigarettes smoked per day. Interestingly, a recent paper that calculated the fraction of attributable risk for HCC associated with smoking as well as viral infection ${ }^{28}$ found that HBV and HCV infections were associated with attributable risks of $13 \%$ and $21 \%$, respectively, whereas smoking carried an attributable risk of almost $50 \%$. Although smoking conveys only a small increase in RR compared with viral infection, its high prevalence leads to a high attributable risk.

A role for smoking in HCC is biologically plausible because several chemicals in tobacco smoke, including 4-aminobiphenyl (4-ABP) and polycyclic aromatic hydrocarbons (PAH), can be metabolized into reactive carcinogens in the liver. We previously developed antibodies that recognize the DNA adducts of 4-ABP and PAH, and used them to quantify adduct levels in liver tissues of patients with HCC and controls from Taiwan. ${ }^{29,30}$ After stratification of the immunoperoxidase staining intensities of 4-ABP-DNA adduct levels into tertiles relative to control tissues, multivariate adjusted ORs for HCC were found to increase monotonically, with ORs of 3.4 (95\% CI $0.8-14.3)$ and 6.48 (95\% CI 1.6-26.5) for medium and high adduct levels, respectively, compared with low adduct levels. These same tissues were also stained for the presence of PAH-DNA adducts and staining intensity was classified by tertile. ORs again increased for medium (OR 2.3, 95\% CI $0.6-$ 9.1) and high (OR 3.9, 95\% CI 1.0-14.9) adduct levels compared with low adduct levels. In addition to these two adducts, $\mathrm{AFB}_{1}$-DNA adducts were also measured. Compared with the combined subjects with low levels of all three adducts or high levels of only one adduct, subjects with high levels of two adducts had an OR of 13.1 (95\% CI 3.1-55.5), while those with elevated levels of all three adducts had the highest risk (OR 36.7; 95\% CI 7.2-187.2). CIs were wide, primarily because of difficulties with obtaining liver tissue from controls; only 37 samples were available compared with 105 from cases. However, these data suggest the importance of multiple exposures in $\mathrm{HCC}$ induction.

$\mathrm{PAH}$ exposure was also measured in the Taiwanese CSP cohort described above, using albumin adducts in blood as a biomarker. $^{31}$ When compared with subjects in the lowest quantile, there was an increased risk of HCC, with adjusted ORs of 1.0 (95\% CI 0.5-2.0), 1.2 (95\% CI 0.6-2.4), and 2.0 (95\% CI 1.0-4.2) ( $\left.P_{\text {trend }}=0.08\right)$ for subjects in the second, third, and fourth quantiles, respectively.

\section{Other chemicals}

Liver angiosarcoma has been reported in workers extensively exposed to vinyl chloride monomer, the gas used in the production of polyvinyl chloride. A follow-up study of more than 1,600 Italian male workers indicated that autoclave workers with the highest exposures to vinyl chloride had an almost 10 -fold increased risk of HCC compared to workers with low or no exposure. ${ }^{32}$

In addition to aflatoxins, alcohol, and vinyl chloride, the IARC lists older high-dose combined oral contraceptives, plutonium, and thorium-232 and its decay products (colloidal thorium dioxide [Thorotrast], used as an angiographic agent) as agents with sufficient evidence in humans to be identified as liver carcinogens (http://monographs.iarc.fr/ENG/ Classification/Table4.pdf). Among the agents the IARC lists as having limited evidence in humans are androgenic steroids, arsenic and inorganic arsenic compounds, betel quid without tobacco, polychlorinated biphenyls, trichloroethylene, and X- and gamma-radiation.

\section{Animal studies of liver carcinogens}

Animal studies have identified a number of compounds that induce liver cancer in rodents; whether these compounds also induce HCC in humans is unclear. Wogan has reviewed genotoxic and non-genotoxic animal liver carcinogens. ${ }^{33}$ The genotoxic animal carcinogens for which there is potential human exposure include mycotoxin contaminants of food 
crops (aflatoxins $B_{1}, G_{1}$, and $M_{1}$; ochratoxin $A$ ), a toxin produced by cyanobacteria (blue-green algae) in polluted drinking water (microcystin-LR), and constituents of plants used for food or medicine (cycasin [methylazoxymethanol], alkylbenzene derivatives [safrole, estragole, methyleugenol, beta asarone], and pyrrolizidine alkaloids). The processing and cooking of foods is also known to result in the production liver carcinogens, including $N$-nitroso compounds (dimethylnitrosamine, $\mathrm{N}$-nitrosopyrrolidine) and heterocyclic amines (IQ, MeIQx, amino acid pyrolysis products).

Tamoxifen induces liver tumors in rats but not in mice or hamsters; there are no data suggesting an increased risk for $\mathrm{HCC}$ in women taking this drug. Non-genotoxic rodent-liver carcinogens and tumor promoters to which humans can be exposed include mycotoxins (fumonisins), peroxisome proliferators (trichloroethylene, diethylhexylphthalate, clofibrate), chlorinated hydrocarbons (organochlorine pesticides, e.g. dichlorodiphenyltrichloroethane [DDT], chlordane, pentachlorophenol), polychlorinated biphenyls, 2,3,7,8-tetrachlorodibenzodioxin (TCDD), solvents such as chloroform, and the drug oxazepam. The importance of these chemicals in human HCC risk remains to be determined.

\section{Conclusions}

HBV infection is a well-established risk factor for the development of HCC. Fortunately, a vaccine to prevent infection is available, and has led to a decreased incidence of HCC in countries where universal HBV immunization has been initiated. Data from Taiwan demonstrate an effectiveness rate of $78-87 \%$ for the vaccine, based on the seroprevalence of HBsAg. ${ }^{34}$ Unfortunately, a vaccine for $\mathrm{HCV}$ infection is not currently available, meaning this remains a significant public health problem in the USA. As we learn more about the role of diet, lifestyle, and chemical exposure in HCC development, there will be additional opportunities to intervene and prevent this often fatal disease.

\section{Conflict of interest}

None

\section{Author contributions}

Literature review (HCW), manuscript writing (RMS and $\mathrm{HCW}$ ).

\section{Acknowledgments}

This work was supported by National Institutes of Health grants CA013696, ES005116, and ES009089.

\section{References}

[1] Ferlay J, Shin HR, Bray F, Forman D, Mathers C, Parkin DM. Estimates of worldwide burden of cancer in 2008: GLOBOCAN 2008. Int J Cancer 2010; 127:2893-2917.

[2] El-Serag HB, Rudolph KL. Hepatocellular carcinoma: epidemiology and molecular carcinogenesis. Gastroenterology 2007; 132:2557-2576.

[3] Siegel R, Naishadham D, Jemal A. Cancer statistics, 2012. CA Cancer ] Clin 2012;62:10-29.

[4] Kew M. Epidemiology of chronic hepatitis B virus infection, hepatocellular carcinoma, and hepatitis B virus-induced hepatocellular carcinoma. Pathol Biol 2010;58:273-277.
[5] Bosch FX, Ribes J, Díaz M, Cléries R. Primary liver cancer: worldwide incidence and trends. Gastroenterology 2004;127:S5-S16.

[6] Kensler TW, Roebuck BD, Wogan GN, Groopman JD. Aflatoxin: a 50-year odyssey of mechanistic and translational toxicology. Toxicol Sci 2011;120 (Suppl 1):S28-S48.

[7] Zhang Y], Rossner P, Chen $Y$, Agrawal M, Wang $Q$, Wang L, et al. Aflatoxin $B_{1}$ and polycyclic aromatic hydrocarbon adducts, p53 mutations and p16 methylation in liver tissue and plasma of hepatocellular carcinoma patients. Int J Cancer 2006;119:985-991.

[8] Zhang YJ, Chen C], Lee CS, Haghighi B, Yang GY, Wang LW, et al. Aflatoxin $B_{1}$-DNA adducts and hepatitis $B$ virus antigens in hepatocellular carcinoma and non-tumorous liver tissue. Carcinogenesis 1991;12:2247-2252.

[9] Wu HC, Santella R. The role of aflatoxins in hepatocellular carcinoma. Hepat Mon 2012;12:e7238.

[10] Hatch MC, Chen CJ, Levin B, Ji BT, Yang GY, Hsu SW, et al. Urinary aflatoxin levels, hepatitis-b virus infection and hepatocellular carcinoma in Taiwan. Int J Cancer 1993;54:931-934.

[11] Ross RK, Yu MC, Henderson BE, Yuan JM, Qian GS, Tu JT, et al. Urinary aflatoxin biomarkers and risk of hepatocellular carcinoma. Lancet 1992;339: 943-946.

[12] Qian GS, Ross RK, Yu MC, Yuan JM, Gao YT, Henderson BE, et al. A follow-up study of urinary markers of aflatoxin exposure and liver cancer risk in Shanghai, People's Republic of China. Cancer Epidemiol Biomarkers Prev 1994;3:3-10.

[13] Chen C, Wang L, Lu S, Wu M, You S, Zhang Y, et al. Elevated aflatoxin exposure and increased risk of hepatocellular carcinoma. Hepatology 1996; 24:38-42.

[14] Wang LY, Hatch M, Chen CJ, Levin B, You SL, Lu SN, et al. Aflatoxin exposure and risk of hepatocellular carcinoma in Taiwan. Int J Cancer 1996;67:620625.

[15] Sun CA, Wang LY, Chen CJ, Lu SN, You SL, Wang LW, et al. Genetic polymorphisms of glutathione $S$-transferases $\mathrm{M} 1$ and $\mathrm{T} 1$ associated with susceptibility to aflatoxin-related hepatocarcinogenesis among chronic hepatitis B carriers: a nested case-control study in Taiwan. Carcinogenesis 2001;22:1289-1294.

[16] Wu HC, Wang Q, Yang HI, Ahsan H, Tsai WY, Wang LY, et al. Aflatoxin $B_{1}$ exposure, hepatitis $B$ virus infection, and hepatocellular carcinoma in Taiwan. Cancer Epidemiol Biomarkers Prev 2009;18:846-853.

[17] Chen C, Yu M, Liaw Y, Wang L, Chiamprasert S, Matin F, et al. Chronic hepatitis B carriers with null genotypes of glutathione S-transferase M1 and $\mathrm{T} 1$ polymorphisms who are exposed to aflatoxin are at increased risk of hepatocellular carcinoma. Am J Hum Genet 1996;59:128-134,

[18] Yu MW, Lien JP, Chiu YH, Santella RM, Liaw YF, Chen CJ. Effect of aflatoxin metabolism and DNA adduct formation on hepatocellular carcinoma among chronic hepatitis B carriers in Taiwan. J Hepatol 1997;27:320-330.

[19] Sun Z, Lu P, Gail MH, Pee D, Zhang Q, Ming L, et al. Increased risk of hepatocellular carcinoma in male hepatitis B surface antigen carriers with chronic hepatitis who have detectable urinary aflatoxin metabolite $M_{1}$. Hepatology 1999;30:379-383.

[20] Liu Y, Wu F. Global burden of aflatoxin-induced hepatocellular carcinoma: a risk assessment. Environ Health Perspect 2010;118:818.

[21] Johnson NM, Qian G, Xu L, Tietze D, Marroquin-Cardona A, Robinson A, et al. Aflatoxin and PAH exposure biomarkers in a U.S. population with a high incidence of hepatocellular carcinoma. Sci Total Environ 2010;408:60276031.

[22] Hoque A, Patt YZ, Yoffe B, Groopman JD, Greenblatt MS, Zhang YJ, et al. Does aflatoxin $B_{1}$ play a role in the etiology of hepatocellular carcinoma in the United States? Nutr Cancer 1999;35:27-33.

[23] IARC. IARC Monographs. Volume 44. Alcohol Drinking. IARC Press, 1988.

[24] McKillop IH, Schrum LW. Role of alcohol in liver carcinogenesis. Semin Liver Dis 2009;29:222-232.

[25] McGlynn KA, London WT. Epidemiology and natural history of hepatocellular carcinoma. Best Pract Res Clin Gastroenterol 2005;19:3-23.

[26] Donato F, Tagger A, Gelatti U, Parrinello G, Boffetta P, Albertini A, et al. Alcohol and hepatocellular carcinoma: the effect of lifetime intake and hepatitis virus infections in men and women. Am J Epidemiol 2002;155:323-331.

[27] Lee YC, Cohet C, Yang YC, Stayner L, Hashibe M, Straif K. Meta-analysis of epidemiologic studies on cigarette smoking and liver cancer. Int J Epidemiol 2009;38:1497-1511.

[28] Trichopoulos D, Bamia C, Lagiou P, Fedirko V, Trepo E, Jenab M, et al. Hepatocellular carcinoma risk factors and disease burden in a European cohort: a nested case-control study. J Natl Cancer Inst 2011;103:1686-1695.

[29] Wang LY, Chen CJ, Zhang YJ, Tsai WY, Lee PH, Feitelson MA, et al. 4Aminobiphenyl DNA damage in liver tissue of hepatocellular carcinoma patients and controls. Am J Epidemiol 1998;147:315-323.

[30] Chen SY, Wang LY, Lunn RM, Tsai WY, Lee PH, Lee CS, et al. Polycyclic aromatic hydrocarbon-DNA adducts in liver tissues of hepatocellular carcinoma patients and controls. Int J Cancer 2002;99:14-21.

[31] Wu HC, Wang Q, Wang LW, Yang HI, Ahsan H, Tsai WY, et al. Polycyclic aromatic hydrocarbon- and aflatoxin-albumin adducts, hepatitis B virus 
Santella R. M. et al.: Environmental exposures and hepatocellular cancer

infection and hepatocellular carcinoma in Taiwan. Cancer Lett 2007;252: 104-114.

[32] Gennaro V, Ceppi M, Crosignani P, Montanaro F. Reanalysis of updated mortality among vinyl and polyvinyl chloride workers: confirmation of historical evidence and new findings. BMC Public Health 2008;8:21.
[33] Wogan GN. Impacts of chemicals on liver cancer risk. Semin Cancer Biol $2000 ; 10: 201-210$

[34] Chien YC, Jan CF, Huo HS, Chen CJ. Nationwide hepatitis B vaccination program in Taiwan: effectiveness in the 20 years after it was launched. Epidemiol Rev 2006;28:126-135. 